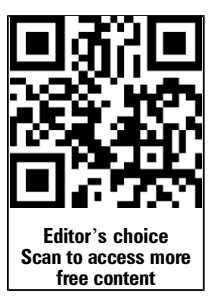

Scan to access mo free conten

\title{
The spectrum of histopathological changes encountered in pancreatectomy specimens after neoadjuvant chemoradiation, including subtle and less-well-recognised changes
}

\author{
Sangeetha N Kalimuthu, Stefano Serra, Neesha Dhani, Runjan Chetty
}

Departments of Pathology, Laboratory Medicine Program and *Medical Oncology, University Health Network and University of Toronto, Toronto, Canada

\section{Correspondence to} Dr Sangeetha Kalimuthu, Department of Pathology, University Health Network, University of Toronto, Toronto General Hospital, 11th floor, Eaton wing, 200 Elizabeth Street, Toronto Ontario, Canada M5G 2C4; kalimuthu.sangeetha@gmail. com

Received 3 January 2016 Revised 26 January 2016 Accepted 2 February 2016 Published Online First 25 February 2016

\begin{abstract}
Preoperative (neoadjuvant) chemoradiation therapy/ treatment (NCRT) is emerging as an important treatment modality in borderline resectable pancreatic ductal adenocarcinoma (PDAC). The constellation of histopathological changes secondary to chemoradiation is diverse and has been well documented, particularly in other gastrointestinal organs such as the oesophagus and colorectum. However, the histological changes specific to the pancreas have not been fully characterised and described. This review aims to provide a detailed catalogue of histological features associated with NCRTtreated PDAC and highlight any subtle, less-recognised changes.
\end{abstract}

\section{INTRODUCTION}

Pancreatic ductal adenocarcinoma (PDAC) is among the most aggressive cancers with approximately 5-6\% 5-year survival rate. At present, surgery offers the best chance of survival; however, only 20\% of PDACs are resectable. Preoperative (neoadjuvant) chemoradiation therapy/treatment (NCRT) is emerging as an important treatment modality in borderline resectable PDAC (tumour abutment or encasement of superior mesenteric vein in the absence of vessel occlusion or extension to the superior mesenteric artery) with the aim to downstage the cancer and improve resectability. At present, chemoradiation for PDACs is more frequently offered in larger centres; however, it is now beginning to be deployed more ubiquitously. Therefore, pathologists can expect to see an increase in NCRT-treated pancreatic resections in routine practice.

In our own institution, we have received approximately 75 cases during the course of the last 10 years and this number continues to increase steadily as more cases received NCRT. As noted in previous studies and in our own institutional experience, different chemoradiation protocols have been trialled in PDAC and these include 5fluorouracil, paclitaxel, gemcitabine administered together with external-beam radiation ranging from 30 to $50.4 \mathrm{~Gy}^{1-7}$ However, presently gemcitabinebased chemoradiation therapy is the preferred preoperative treatment option.

The constellation of histopathological changes secondary to chemoradiation is highly variable and has been well documented in other gastrointestinal organs such as the oesophagus and colorectum..$^{8-12}$ However, the histological changes specific to the pancreas have not been fully characterised. The aim of this review is to provide a detailed evaluation of histological features associated with NCRT-treated PDAC and highlight subtle, less-recognised changes seen, based on our institutional experience gleaned over the last 10 years with such cases. Herein, we outline the full range of specific histological features associated with NCRT-treated PDAC in an effort to make the diagnostic pathologist aware of changes likely to be encountered and potential histopathological pitfalls.

\section{HISTOLOGICAL CHANGES FOLLOWING NEOADJUVANT CHEMORADIATION}

Chemoradiation can affect the morphological appearance of both the tumour and non-neoplastic pancreatic parenchyma, resulting in recognisable histological changes associated with tumour regression. However, occasionally the changes yielded may result in a diagnostic conundrum and/or are less well known. The full constellation of features observed are discussed in greater detail below and summarised in table 1 .

\section{NCRT changes in residual tumour}

The well-recognised treatment-related histological hallmarks were seen in varying proportions in all cases. These included the characteristic cytomorphological changes of nuclear hyperchromasia, pyknosis, irregular/bizarrely shaped nuclei with voluminous, vacuolated and densely eosinophilic cytoplasm (figure 1A). Most tumours were accompanied by varying degrees of coagulative and ischaemic necrosis (figure 1C,D), which was often patchily/focally distributed. Sparse to moderate immediately peritumoural mixed acute and chronic inflammation was seen admixed in the majority of tumours, while intratumoural inflammation was rarely observed and when present was usually acute. The constituents of the inflammatory infiltrate were predominantly lymphoplasmacytic, while neutrophils were seen mainly in the presence of necrosis.

In all cases, the prevailing and appreciable feature was that of tumour being disrupted and dissociated by streams of intervening fibrosis, resulting in small islands and single cells being strewn slightly haphazardly within the stroma (figure 1D). Occasionally, one or more of the following typical regression-associated features-pools of mucin (figure 2A) containing foamy macrophages, foreign body-type giant cells, cholesterol clefts and stromal/ 


\section{Review}

Table 1 Histological changes following preoperative (neoadjuvant) chemoradiation in pancreatic ductal adenocarcinoma

\begin{tabular}{|c|c|c|}
\hline & Common histological features & Uncommon histological features \\
\hline \multirow[t]{6}{*}{ Tumour } & $\begin{array}{l}\text { Cytoplasmic eosinophilia } \\
\text { Hyperchromasia } \\
\text { Pyknosis } \\
\text { Bizarre nuclei }\end{array}$ & $\begin{array}{l}\text { Oncocytic/'oncocyte-like' change } \\
\text { Polygonal cells } \\
\text { Eosinophilic, granular cytoplasm } \\
\text { Round hyperchromatic nuclei } \\
\text { - Cherry red, prominent nuclei }\end{array}$ \\
\hline & $\begin{array}{l}\text { Clear cell change } \\
\text { Wrinkled nuclei } \\
\text { Voluminous clear cytoplasm } \\
\text { 'Lipoblast-like' cells } \\
\text { 'Signet ring-like' cells }\end{array}$ & $\begin{array}{l}\text { Rhabdoid change } \\
\text { Globular, hyaline intracytoplasmic inclusion } \\
\text { Highlighted by cytokeratin staining } \\
\text { Also known as 'cytokeratin aggresomes' }\end{array}$ \\
\hline & $\begin{array}{l}\text { Tumours accompanied by } \\
\text { Mucin pools } \\
\text { Foamy macrophages } \\
\text { Foreign body-type giant cells }\end{array}$ & Squamous metaplasia/differentiation \\
\hline & Tumour dissociated into single cells and small islands by streams of fibrosis & $\begin{array}{l}\text { Biphasic appearance (seen in one case only) } \\
\text { Oncocytic ductal component } \\
\text { 'Endocrine' component (microglandular and organoid patterns) } \\
\text { Negative for neuroendocrine/hormonal markers }\end{array}$ \\
\hline & $\begin{array}{l}\text { Necrosis } \\
\text { Coagulative (ischaemic) type } \\
\text { Tumour necrosis } \\
\text { Peritumoural inflammation } \\
\text { Lymphocytes } \\
\text { Plasma cells } \\
\text { Neutrophilc }\end{array}$ & $\begin{array}{l}\text { Tumour mimicking islet cells } \\
\text { 'Single-filing' pattern } \\
\text { Erythrophagocytosis } \\
\text { Whole red blood cells } \\
\text { Fragmented (effete) red blood cells }\end{array}$ \\
\hline & & $\begin{array}{l}\text { Intracytoplasmic sharply circumscribed lipid droplet } \\
\text { Tumour colonising islet cell aggregates } \\
\text { 'Cookie-cutter' appearance } \\
\text { 'Jigsaw-puzzle' like appearance }\end{array}$ \\
\hline Stroma and vessels & $\begin{array}{l}\text { Stromal fibrosis } \\
\text { Fibromyxoid stroma with 'shredded carrot' appearance } \\
\text { Dense 'keloidal' stroma } \\
\text { Intimal proliferation of medium-sized vessels }\end{array}$ & $\begin{array}{l}\text { Keloidal fibrosis resembling 'amyloid' } \\
\text { Congo red negative }\end{array}$ \\
\hline \multirow[t]{3}{*}{ Non-neoplastic pancreas } & $\begin{array}{l}\text { Pancreatic islet cells } \\
\text { Islet cell hyperplasia } \\
\text { 'Single-file' configuration }\end{array}$ & Marked islet cell hyperplasia mimicking endocrine 'microadenoma' \\
\hline & $\begin{array}{l}\text { Distorted benign ducts, simulating invasive tumour } \\
\text { Hypertrophic nerves }\end{array}$ & $\begin{array}{l}\text { Perineural localisation of islet cells imitating perineural invasion } \\
\text { Benign ducts with cytoplasmic eosinophilia }\end{array}$ \\
\hline & & Benign ducts with squamous metaplasia \\
\hline
\end{tabular}

Figure 1 (A) Tumour with bizarrely shaped nuclei, prominent nucleoli with voluminous, vacuolated and densely eosinophilic cytoplasm $(\times 400$ magnification). ( $B$ and $C$ ) Tumour with large and patchy, geographic areas of coagulative, ischaemic necrosis $(\times 25$ magnification). (D) Intervening fascicular streams of fibrosis resulting in tumour disruption and dissociation. ( $\times 50$ magnification). All H\&E.

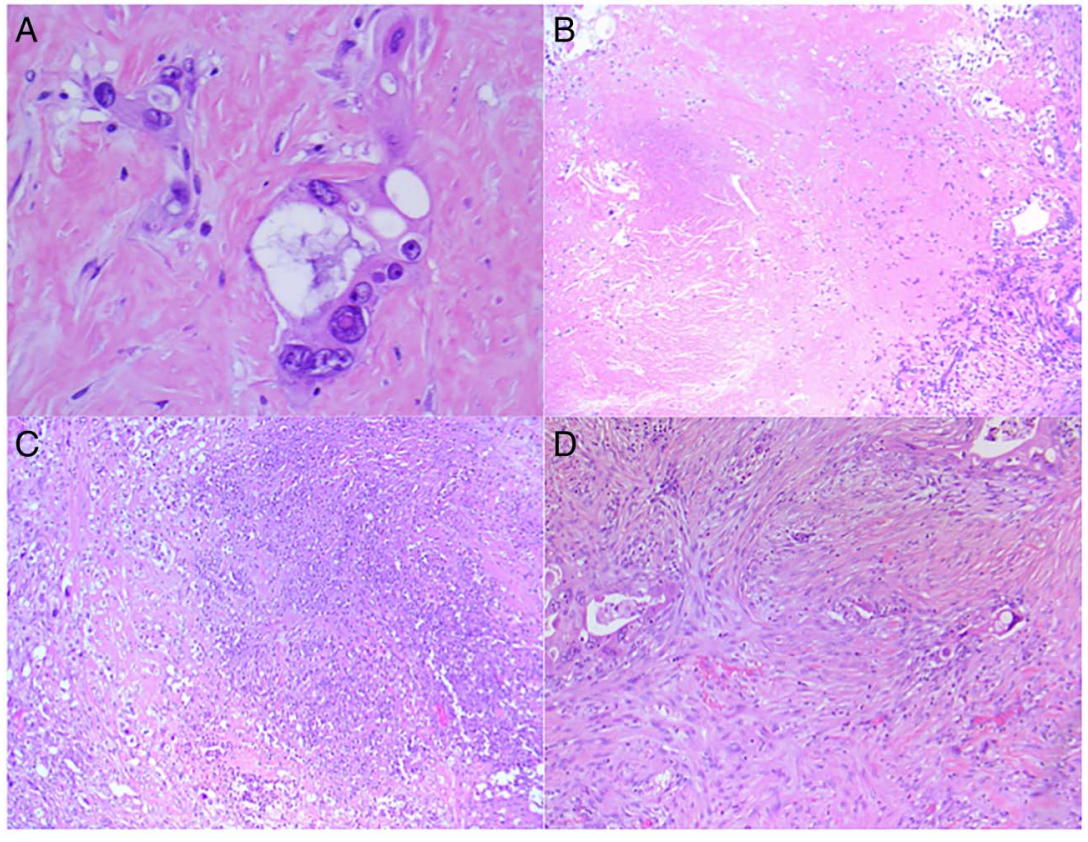


Figure 2 Tumour regression-associated features including (A) tumour disruption by pools of mucin ( $\times 100$ magnification) and $(B)$ sheets of foamy histiocytes with scattered foreign body-type giant cells ( $\times 200$ magnification). (C) Minimal residual tumour (arrow), in a background of marked fibrosis and lobular pancreatic atrophy ( $\times 25$ magnification). (D) The marked degree of atypia in the cells lining the ducts is better appreciated at higher power (×200 magnification). All H\&E.

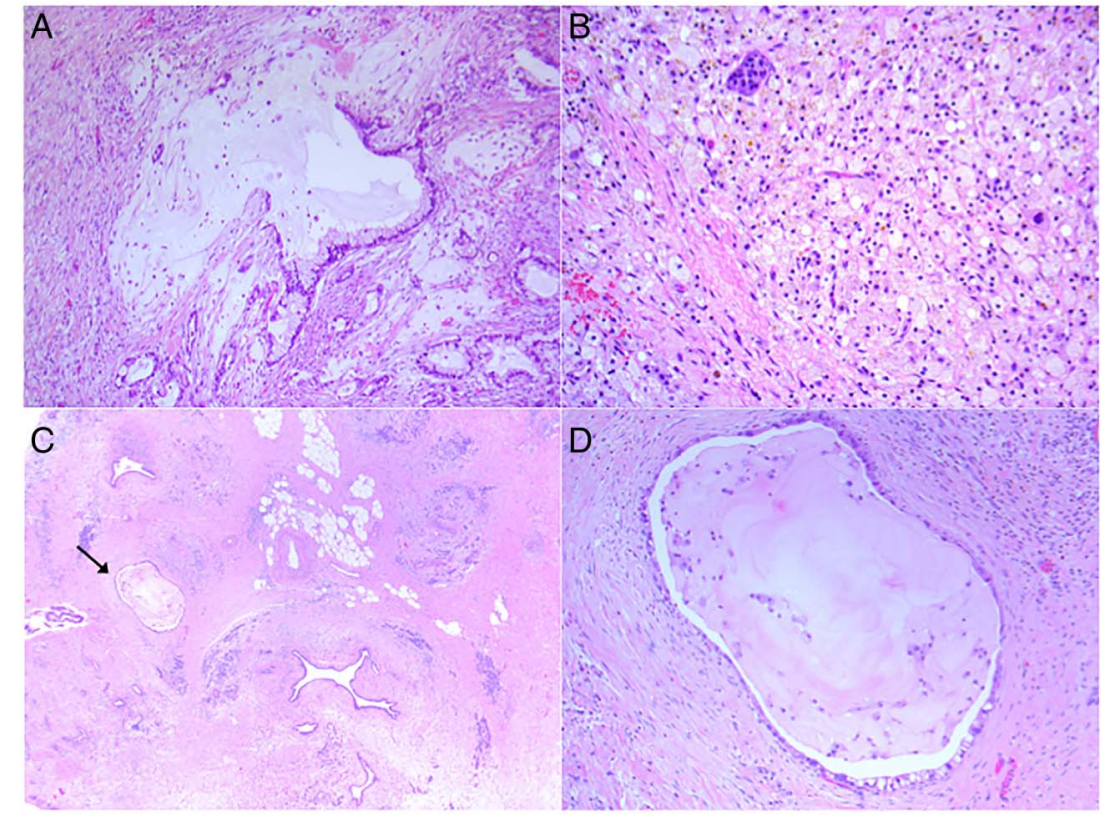

tumoural calcification-accompanied the tumour (figure 2B), although all these features were rarely seen together.

Of note, sparse or minimal residual tumour may sometimes be missed, particularly on low magnification when it blended seamlessly in a milieu of dense fibrosis and surrounding entrapped benign ducts (figure 2C). However, on higher magnification the marked degree of atypia seen within cells lining the duct confirmed the presence tumour and allowed for separation from reactive benign ducts (figure 2D).

Occasionally, the residual tumour was arranged in short cords or rows resembling a 'single-filing' pattern (figure 3A). This appearance sometimes mimicked residual islet cells (figure $3 \mathrm{~B}$ ) or can deceptively colonise a cluster of residual islet cells, resulting in a cribriform configuration, resembling a 'cookie-cutter' or 'jigsaw puzzle-like' appearance (figure 3C). Occasionally, residual tumour can even arrange itself into interlinking, serpentine cords recapitulating the configuration of an islet of Langerhans. Cancer may also be seen expanding and replacing a nerve with only a residual hint of compressed nerve fibres and perineurium (figure 3D).

The tumour displayed a broad spectrum of cytomorphological alterations ranging from clear cell change, cytoplasmic eosinophilia, oncocytic/oncocytic-like change and rhabdoid features. Also, many of these cytoplasmic features were occasionally seen occurring together within the same tumour. Clear cell change was characterised by tubulopapillary glands lined by polygonal to pyramidal in shape (figure 4A) with ample clear, occasionally faintly amphophilic cytoplasm, and irregular, wrinkled nuclei (figure $4 \mathrm{~A}$, inset), which was also frequently noted in concert with either cytoplasmic eosinophilia or oncocytic-like change, where there was a sharp demarcation between the components (figure 4B). Varying amounts of cytoplasmic
Figure 3 ( $A$ and $B$ ) Tumour arranged in short cords and rows resembling a 'single-filing' pattern of pancreatic islets cells $(\times 200$ and 400 magnification). (C) Tumour deceptively colonising residual islet cells resembling a 'cookie-cutter' or 'jigsaw puzzle-like' appearance $(\times 200$ magnification). (D) Interlinking, serpentine cords of tumour recapitulating islet of Langerhans and replacing a nerve with only a residual hint of nerve fibres and perineurium (arrow) (×200 magnification). All H\&E.

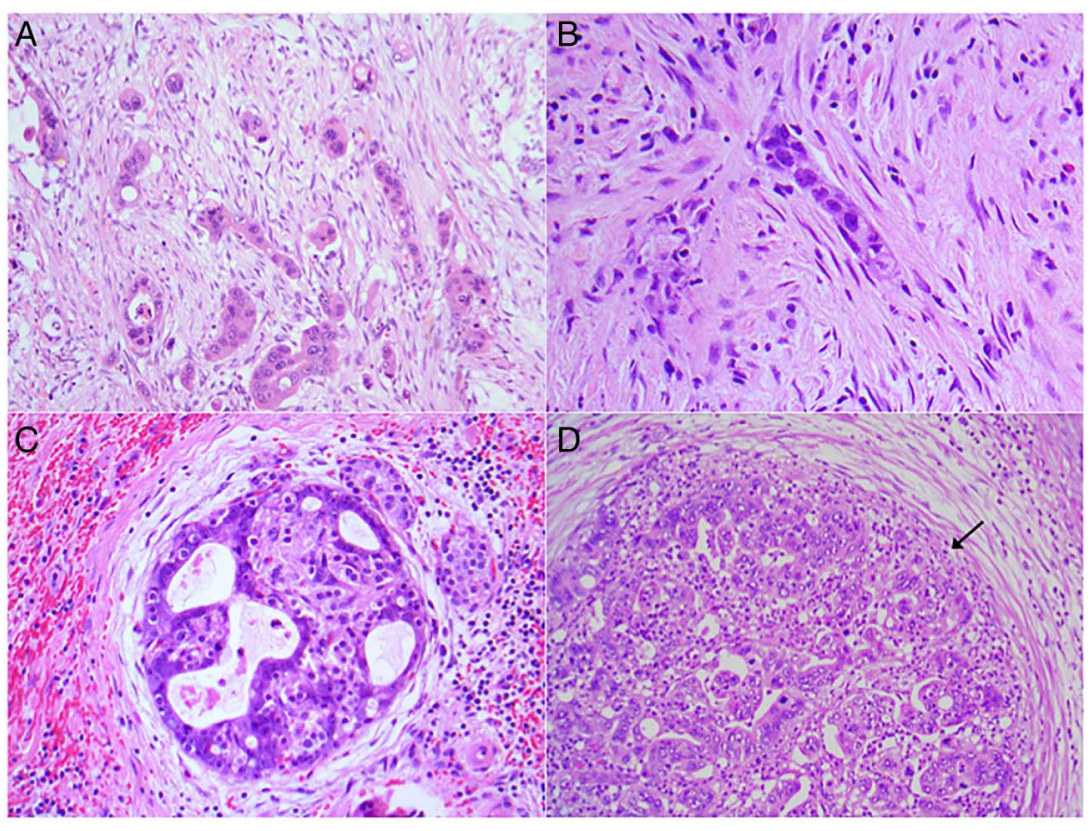


Figure 4 (A) Clear cell change of tumour characterised by large polygonal to pyramidal cells with ample clear to amphophilic cytoplasm, irregular, wrinkled nuclei (inset) $(\times 50$ magnification). (B) Tumour gland shows sharp demarcation between cytoplasmic eosinophilic component and clear cell component $(\times 200$ magnification); clear cell change accompanied by varying patterns of cytoplasmic vacuolation, including (C) single large vacuole displacing the nuclei resembling a signet ring cell (arrow) and (D) multiple coalescing vacuoles resembling 'lipoblasts' (arrow) (×400 magnification). All H\&E.

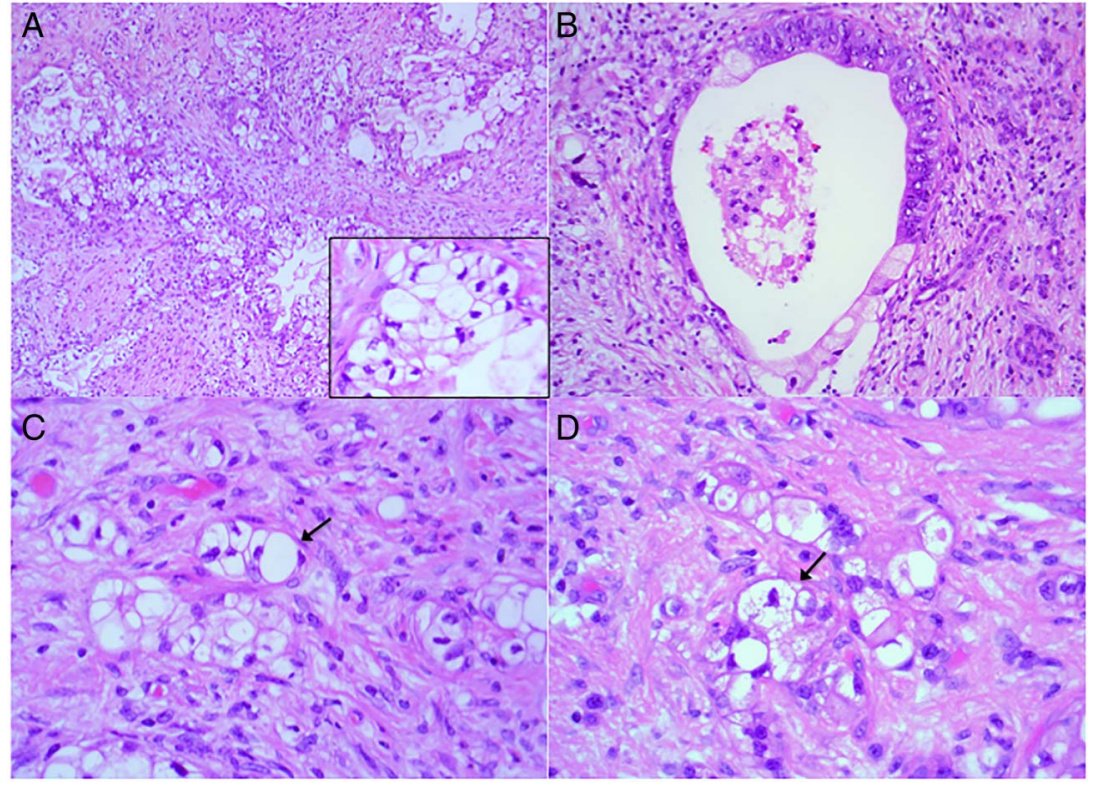

vacuolation were also seen in many of the tumour cells. The pattern of vacuolation ranged from a single large vacuole displacing the nucleus peripherally, thus resembling a signet ring cell (figure 4B), to coalescing, moderately sized vacuoles indenting the nucleus, resembling lipoblasts (figure 4D), and even to multiple tightly packed vacuoles eliciting a frothy, 'lather-like' cytoplasmic appearance. Cytoplasmic eosinophilia was ubiquitously present within tumour cells seen as deeply eosinophilic cytoplasm (figure 5A). Cytoplasmic eosinophilia should be distinguished from true oncocytic/oncocytic-like change, which is characterised by cells assuming a polygonal configuration with voluminous eosinophilic granular cytoplasm, large, centrally located round hyperchromatic nuclei and cherry red, prominent nucleoli (figure 5B). Using the aforementioned definition, true oncocytic change was only seen in a minority of cases. Rhabdoid morphology was observed focally as a minor component, often only featuring scattered cells within the tumour. However, these cells were unmistakable given their distinct appearance characterised by globular, waxy, hyaline intracytoplasmic inclusions with resultant eccentric displacement of the nuclei (figure 5C). Furthermore, keratin stains highlighted the globular staining pattern of the hyaline inclusions (figure 5D), which are also referred to as 'cytokeratin aggresomes'.

One tumour demonstrated an intriguing post-treatment appearance. It appeared as a distinct biphasic tumour comprising a predominantly oncocytic ductal component and a second, more solid hyperchromatic 'endocrine-appearing' component (figure 6A). The oncocytic component showed a variety of architectural patterns with cords and sinuous branching ducts, occasionally coalescing to form loose nodules (figure 6B). The cells were pyramidal to polygonal in shape with large pleomorphic nuclei, dispersed chromatin, prominent nucleoli and
Figure 5 (A) Tumour with evidence of cytoplasmic eosinophilia (H\&E). (B) Oncocytic/oncocytic-like change demonstrating characteristic polygonal cells with voluminous eosinophilic, granular cytoplasm, large, round hyperchromatic nuclei and prominent nucleoli (H\&E). (C) Focal rhabdoid change (arrow) with distinct globular hyaline intracytoplasmic inclusion with eccentric displacement of nuclei (H\&E). (D) Cytokeratin stain highlighting the intracytoplasmic inclusion, also referred to as 'cytokeratin aggresomes' (arrow). All figures $\times 400$ magnification.

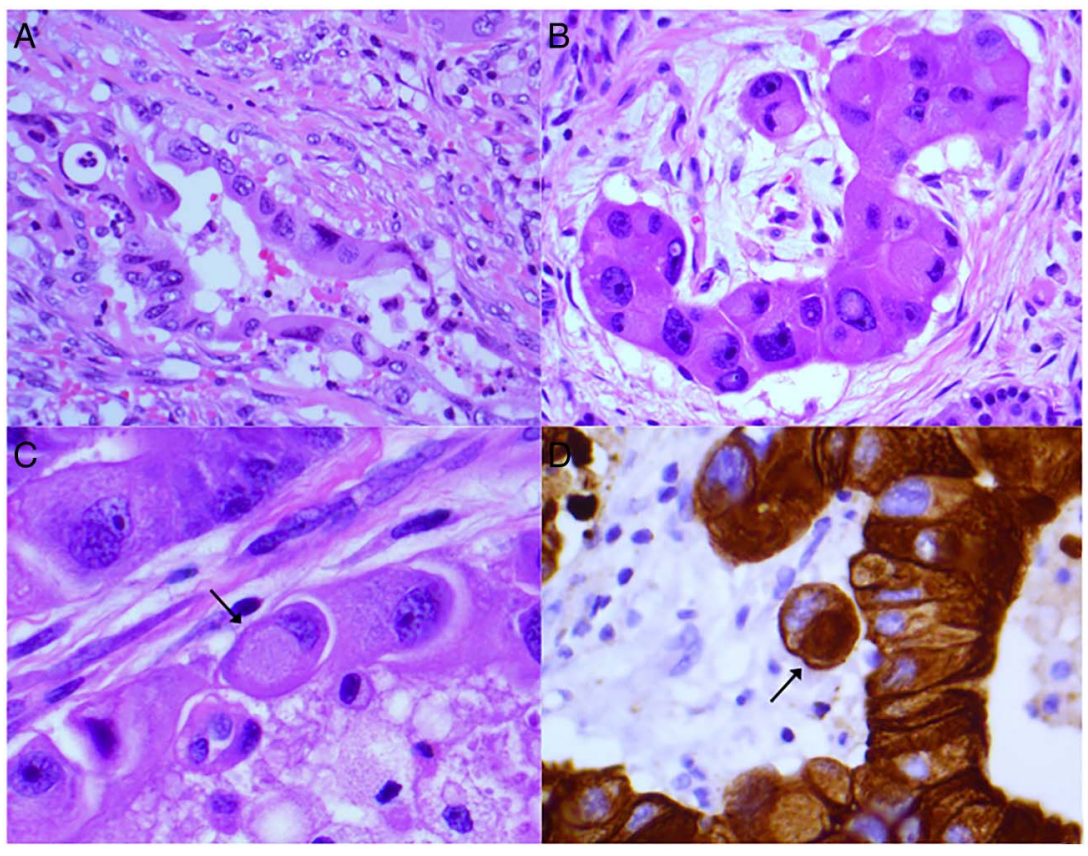


Figure 6 (A) Biphasic tumour comprising oncocytic ductal component (arrowhead) and a hyperchromatic, 'endocrine-appearing' component (arrow) $(\times 25$ magnification). (B) Oncocytic component composed of cords and sinuous branching ducts, focally forming loose nodules $(\times 200$ magnification). Both $\mathrm{H} \& \mathrm{E}$.

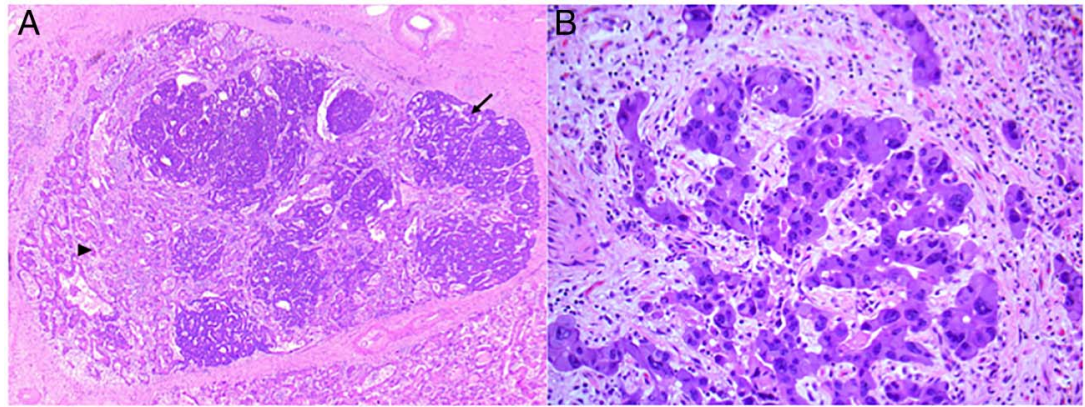

abundant granular eosinophilic cytoplasm (see figure 5B) and occasional glands contained luminal inspissated, eosinophilic material (figure 6B). In the largest nodule, the oncocytic component merged seamlessly with the more hyperchromatic, 'endocrine-like' component (figure 7A). The latter demonstrated two principal histological patterns: a microglandular, gyriform, ribbon-like pattern (figure $7 \mathrm{~B}$ ) and a focal solid, organoid pattern (figure 7C). The cells in this area were smaller, relatively uniform cuboidal in shape with round nuclei, stippled chromatin, prominent nucleoli and moderate amounts of finely granular cytoplasm (figure 7D), resembling resident islet cells. On immunohistochemical workup, both morphological components were found to be positive for cytokeratin 7 (figure 8A) and entirely negative for neuroendocrine markers (figure $8 \mathrm{~B}$ ) as well as specific hormonal markers (insulin, glucagon), resulting in a diagnosis of post-treated residual conventional PDAC.

Rare tumour cells showed evidence of erythrophagocytosis, a well-described phenomenon in cancer cells, incorporating both whole and fragmented (effete) red blood cells (figure 9A) within the cytoplasm.

Lastly, multiple, coalescing, globular, sharply demarcated lipid-like droplets were seen within the cytoplasm of tumour cells vaguely resembling a 'silicone granuloma-like' appearance (figure 9B).

\section{NCRT changes in stroma and vessels}

Stromal fibrosis, whether secondary to tumour desmoplasia, chronic pancreatitis or tumour regression was a consistent and clearly evident feature in all cases. There was considerable variation in stromal cellularity, which was attributed to the different stages of evolution of the fibrosis. The fibrosis seen in our series can be broadly divided into three distinct appearances, which are as follows:

1. Loose, paucicellular, variably fibrotic and myxoid stroma comprising delicate stellate fibroblastic cells and scattered, prominent wavy fibrous bundles, resulting in a 'shredded-carrot' appearance (figure 10A).

2. Hypocellular, dense keloidal fibrosis with scattered delicate fibroblasts (figure 10B).

3. Moderately to markedly cellular stroma comprising haphazard fascicles of plump, pleomorphic fibroblasts with irregular nuclei, granular chromatin and tapering cytoplasmic processes, occasionally resembling nodular fasciitis with accompanying granulation tissue formation (figure 10C). The keloidal-type fibrosis occasionally mimicked the appearance of amyloid although lacking the characteristic amorphous quality of amyloid (figure 10D).

The inflammatory component varied from sheets of foamy macrophages incorporating foreign body-type giant cells (see figure 2C) to mixtures of plasma cells, lymphocytes, eosinophils and histiocytes admixed with and scattered throughout the fibrous stroma (figure 11A). In several cases, haemosiderin deposits were seen patchily distributed throughout the stroma. Vascular changes consisted of myxohyaline intimal proliferation that was seen in the majority of medium-sized vessels (figure 11B).
Figure 7 (A) Biphasic tumour shows both the oncocytic and 'endocrine-like' components seamlessly merging together ( $\times 100$ magnification); 'endocrine-like' component with two morphological patterns. (B) Microglandular, gyriform, ribbon-like pattern and $(C)$ more solid, organoid pattern ( $\times 200$ magnification). (D) The cells are relatively uniform with round nuclei, stippled chromatin and finely granular cytoplasm $(\times 400$ magnification). All H\&E.

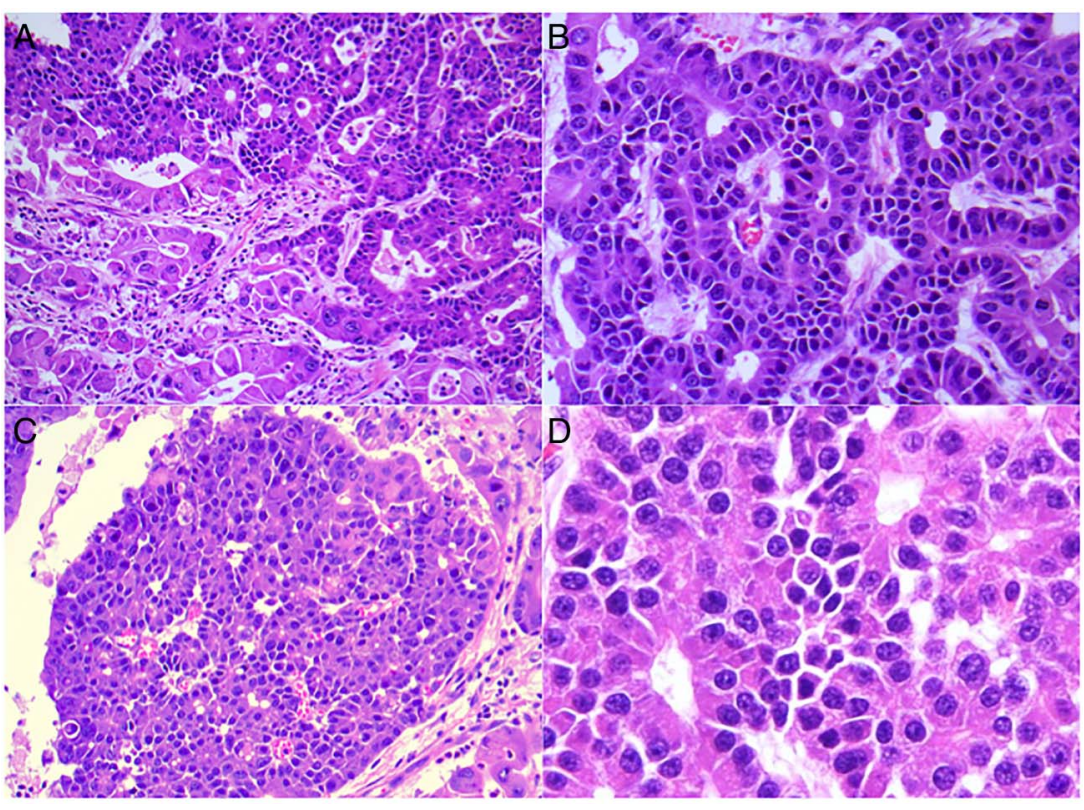


Figure 8 (A) The entire tumour is positive for cytokeratin $7(\times 25$ magnification). (B) All neuroendocrine markers (chromogranin shown here) only decorate residual islet cells (arrow) (×50 magnification). are entirely negative in the tumour and

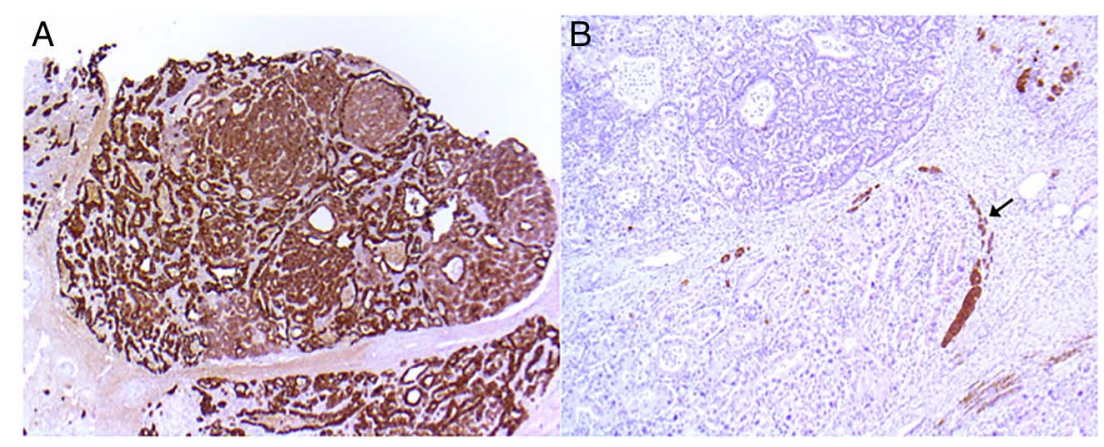

Figure 9 (A) Tumour cells show evidence of erythrophagocytosis, incorporating both whole (arrow) and fragmented/effete red blood cells (arrowhead). (B) 'Silicone granuloma-like' appearance of tumour as a result of multiple, globular, sharply demarcated cytoplasmic lipid-like droplets. Both $\times 400$ magnification, H\&E.

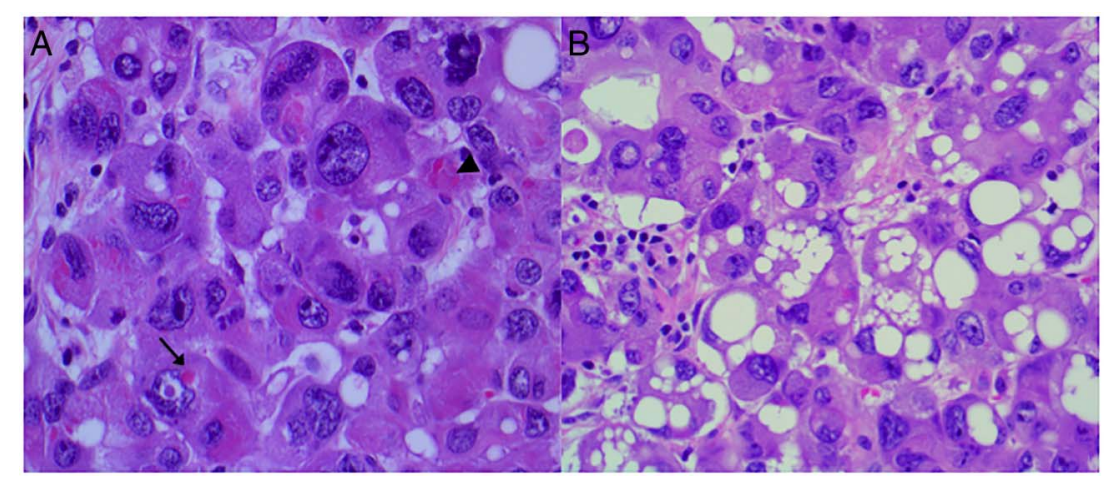

\section{NCRT changes in non-neoplastic pancreas}

There was extensive lobular atrophy and loss of pancreatic acini as a result of fibrous replacement. There was florid islet cell hyperplasia and aggregation in all cases in a similar fashion to that seen in chronic pancreatitis. Typically, small nests and cords of endocrine cells were seen to dissociate from residual enlarged islet cell aggregates and trail off into the surrounding fibrotic stroma. In this setting, the islet cell cords commonly assumed a 'single file' (figure 12A) and additionally were seen to be intimately associated with fibrosis and benign ducts, mimicking invasive tumour (figure 12B). In addition, islet cells exhibited perineural localisation, masquerading as tumour perineural invasion (figure 12C). In one case, the marked hyperplasia of the islet cell aggregate resulted in a distinct trabecular pattern, mimicking an endocrine microadenoma (figure 12D).

Residual benign ducts can occasionally be distorted by the surrounding fibrosis and ductal epithelium can display a mild degree of chemoradiation-induced atypia (figure 13A), which can be misinterpreted as invasive tumour. It is also worth noting the cytoplasmic eosinophilia observed in tumour cells can also be appreciated in benign ductal epithelium (figures 13B).

Squamous metaplasia of invasive cancer as well as benign ducts was occasionally observed (figures 13C,D). Groups of
Figure 10 (A) Fibromyxoid stroma with scattered wavy fibrous bundles, resembling a 'shredded-carrot' appearance ( $\times 100$ magnification). (B) Hypocellular dense 'keloidal' stroma (×100 magnification). (C) Markedly cellular stroma with haphazard fascicles of pleomorphic fibroblasts, resembling nodular fasciitis $(\times 50$ magnification). (D) Irregular, twisted bundles of 'keloidal' stroma (arrow), firmly embedded within a hyperplastic islet cell aggregate, mimicking amyloid deposition. (×200 magnification). All H\&E.

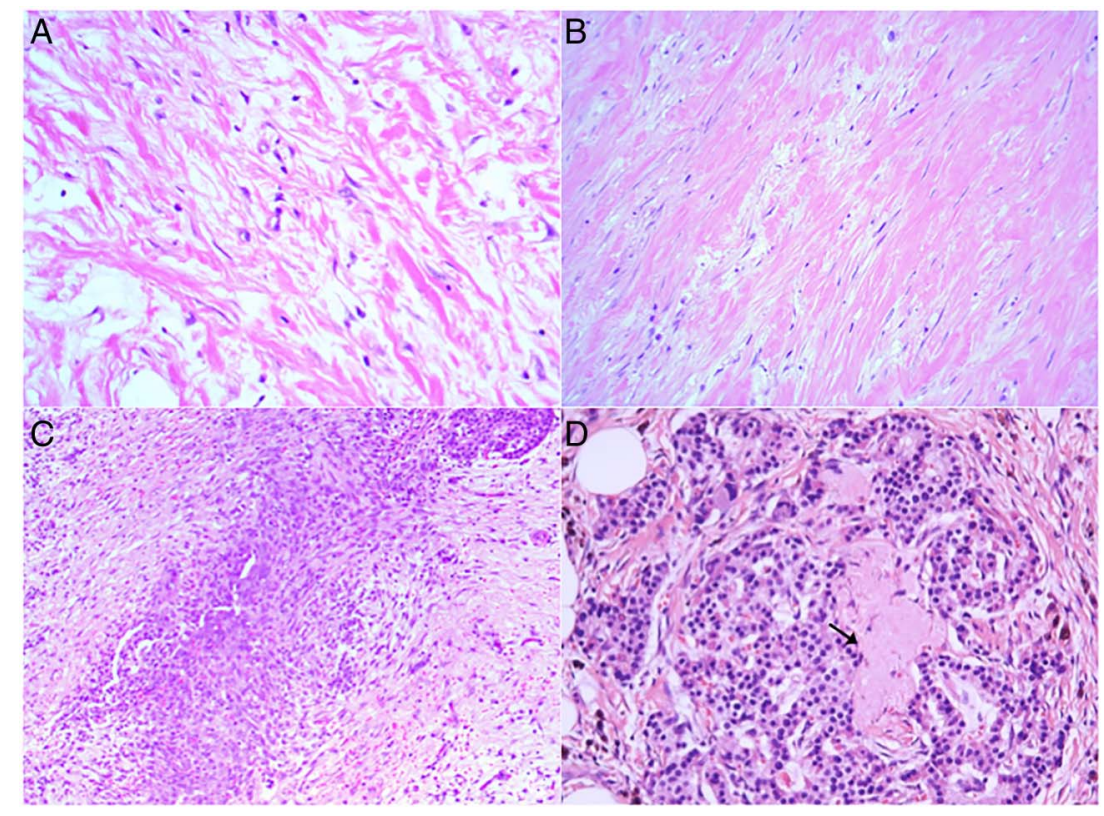


Figure 11 (A) Stromal and peritumoural inflammation composed of a mixture of plasma cells, lymphocytes and eosinophils $(\times 100$ magnification). (B) Medium-sized vessel shows myxohyaline intimal proliferation (arrow) $(\times 100$ magnification). Both $\mathrm{H} \& \mathrm{E}$.

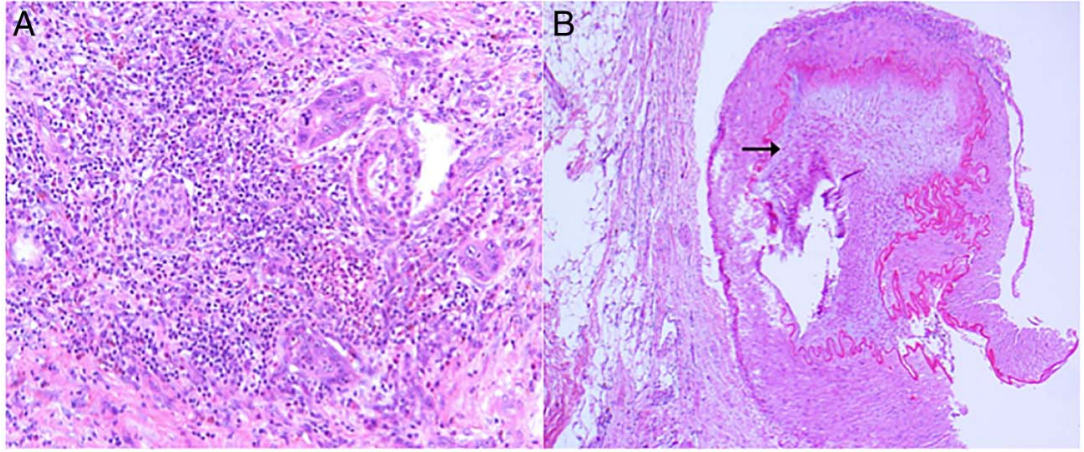

haphazardly distributed hypertrophic nerve bundles were present in all cases, especially in the peripancreatic tissue.

\section{DISCUSSION}

The effects of chemoradiation can induce a panoply of changes in both the neoplastic and non-neoplastic pancreas, which can result in potential diagnostic pitfalls. When there was a relative paucity of tumour, it was often difficult to distinguish invasive tumour from benign ducts as the latter was frequently distorted by the marked accompanying fibrosis. This interpretative difficulty was further compounded when benign ducts showed a mild degree of atypia as a result of treatment effect. In addition, dissociated fragments of residual tumour mimicked residual islet cells by adopting a 'single file' arrangement and could also be seen to colonise residual islet cells. The latter features can be subtle and may be missed at first glance. In contrast, the residual normal islet cells illusively mimicked invasive tumour, particularly when embedded in the stroma as single cells or when intimately associated with benign ducts. Furthermore, these islet cells were also seen to localise in the perineural space, resembling perineural invasion. Bartow et al also frequently observed this feature in the setting of pancreatic fibrosis post-chronic pancreatitis. $^{13}$

The islet cell hyperplasia can be quite striking and can aggregate to reach a large size. ${ }^{14}$ It is important that the latter not be mistaken for an endocrine microadenoma. This conundrum can be circumvented with the knowledge that islet cell hyperplasia, in comparison to endocrine microadenomas, is often closely associated with numerous other islet cell aggregates of varying sizes (as illustrated here; see figure 11D), more commonly seen in a background of chronic pancreatitis and retains expression of all four normal islet peptides (insulin, glucagon, somatostatin and pancreatic polypeptide). ${ }^{15}$

The main cytomorphological appearances (cytoplasmic eosinophilia, oncocyte-like change and clear cell change) were observed consistently in all tumours. Occasionally, all three features were seen focally within the same tumour; however, cytoplasmic eosinophilia was most frequently observed.

Cytoplasmic eosinophilia is a well-established chemoradiation-related change and has been well documented in a spectrum of tumours including the oesophagus, ${ }^{81617}$ head and neck, lung ${ }^{18}$ and colorectum. ${ }^{12} 1719$ This increased eosinophilia in the cells is regarded as one of a group of changes serving as the diagnostic indication of irreversible cellular injury, ${ }^{1} 1820$ which occurs in unison with one or more of the following nuclear features: nuclear hyperchromasia, pyknosis, karyorrhexis or karyolysis. Of note, recognition of these features forms the basis of the regression grading system proposed by Evans et $a l .{ }^{1}$ As such, it can be presumed that the eosinophilia is a degenerative feature directly induced by chemoradiation.

The oncocyte-like change differs from the cytoplasmic eosinophilia in that they are larger with more voluminous
Figure 12 (A) Islet cells arranged in cords resembling a 'single-file' pattern (×200 magnification). (B) Irregular groups and cords of islet cells intimately associated with fibrosis and benign ducts, simulating invasive tumour ( $\times 100$ magnification). (C) Islet cells with perineural localisation (arrow), masquerading as perineural invasion ( $\times 400$ magnification). (D) Large islet cell aggregate with a trabecular pattern, which may be mistaken for an endocrine microadenoma (arrow) $(\times 25$ magnification). All H\&E.

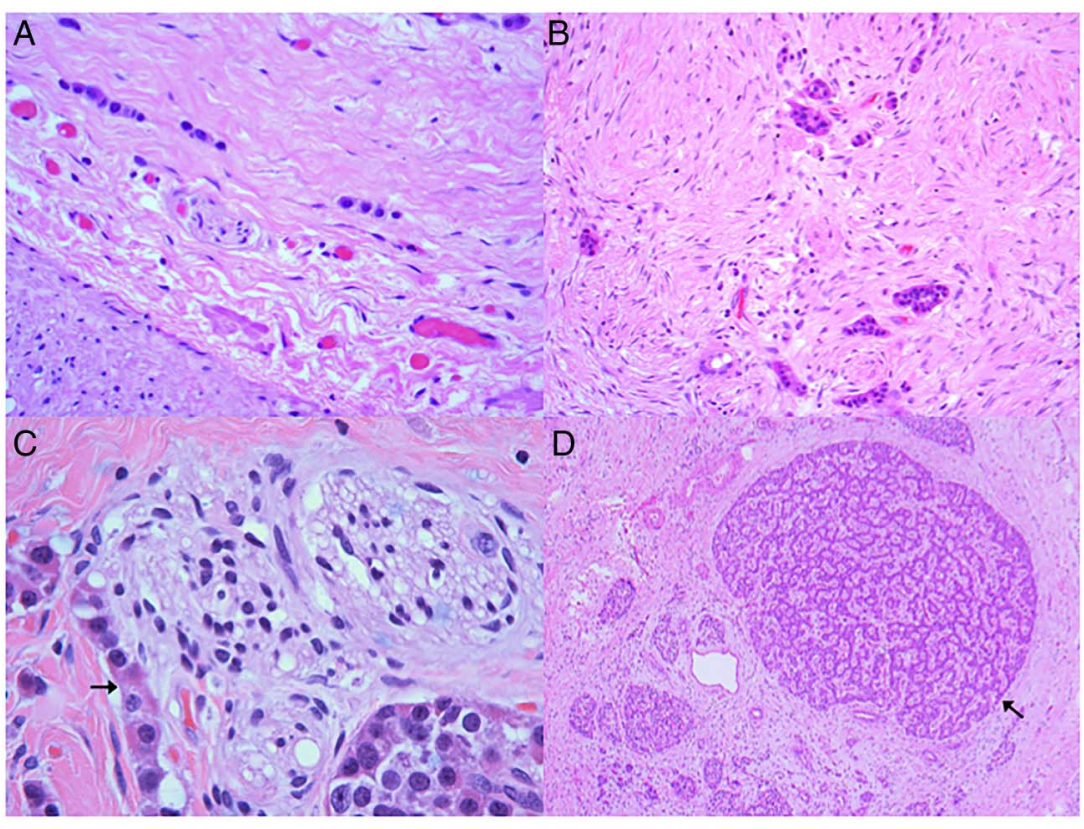


Figure 13 (A) Irregular, distorted benign ducts resembling invasive tumour ( $\times 50$ magnification). (B) Cytoplasmic eosinophilia can also be observed in benign ducts $(\times 200$ magnification). (C) Squamous metaplasia in invasive tumour and (D) benign ducts ( $\times 200$ magnification). All H\&E.

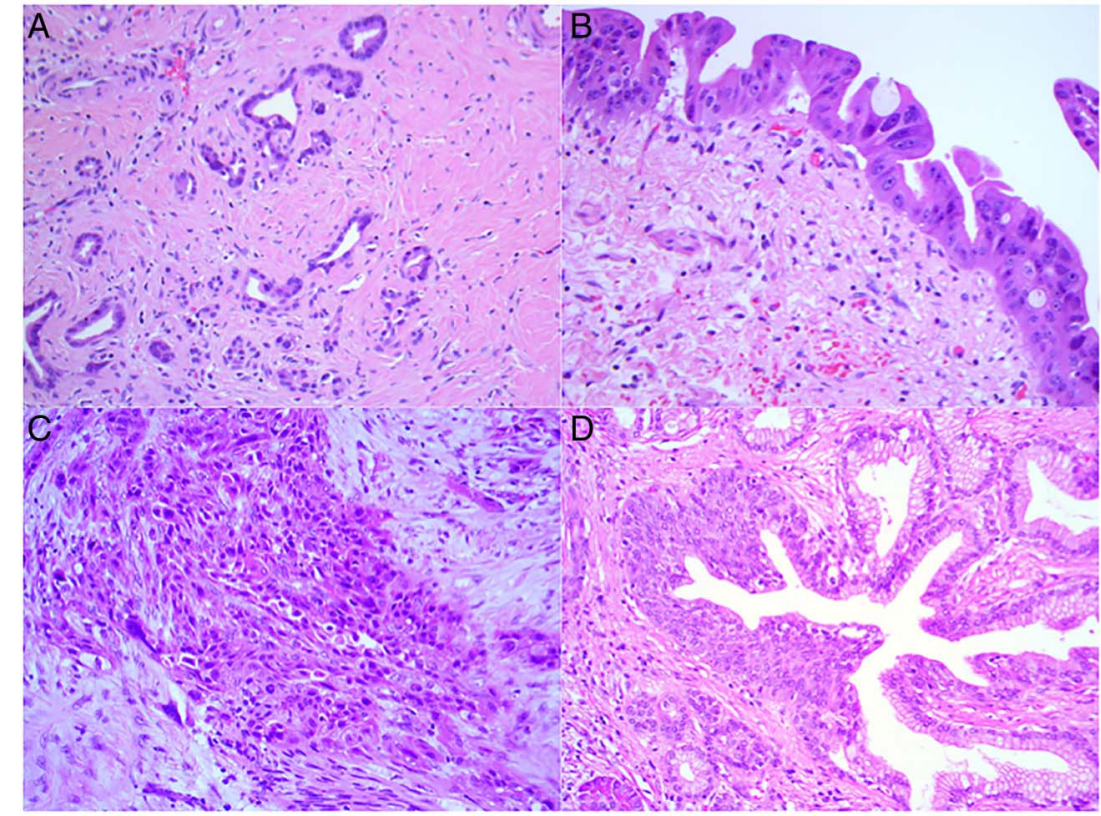

granular cytoplasm, with a faintly shimmering quality. The accumulation of mitochondria in the cytoplasm gives these cells its characteristic appearance. ${ }^{20}$ The precise aetiology of this oncocytic change in the setting of chemoradiation is unknown and remains somewhat speculative. ${ }^{21-23}$ Shia et al ${ }^{2425}$ demonstrated the presence of a significant number of eosinophilic cells with endocrine differentiation in chemoradiation-treated rectal adenocarcinomas. This was supported by chromogranin positivity, suggesting this line of differentiation to be an advanced form of treatment response. However, in a study carried out by Rouzbahman et al, ${ }^{21}$ the five cases of post-treated rectal adenocarcinomas with oncocytic change showed no evidence of endocrine/neuroendocrine features, proposing that this change may be either a degenerative process or cell response to hypoxia secondary chemoradiation.

The interesting unique case discussed earlier raises an interesting conjectural issue. Given the strong morphological resemblance to 'endocrine' cells, could this be a post-treatment effect where the carcinoma is assuming a neuroendocrine phenotype although lacking the immunophenotype? Neuroendocrine differentiation post-hormone treatment in prostatic carcinoma has been described; however, the carcinomas displayed positive neuroendocrine markers. ${ }^{26}$ The other diagnostic possibilities explored, given the biphasic morphological appearance of the tumour, included a mixed acinar-endocrine carcinoma of the pancreas $^{27}$ or a mixed acinar endocrine ductal carcinoma. However, again, the absence of staining for neuroendocrine and other specific hormonal markers refuted this diagnosis. Both the aforementioned tumours are prototypical examples of cases that give weight to the assertion that exocrine, endocrine and perhaps even ductal elements of the pancreas share a common embryologic origin. Using mouse models, a recent study has shown that Sox-9 (a member of the Sry-related high mobility group box transcription factors)-expressing cells are present throughout the biliary and pancreatic ductal epithelium, which are connected to the intestinal stem cell zone. ${ }^{28}$ They further demonstrated that embryonic pancreatic Sox-9-expressing cells differentiate into all mature cell types; however, their capacity for endocrine differentiation declines shortly after birth as these endocrine cells detach from the ductal epithelial lining and form pancreatic islet cells. It is also worth mentioning here another controversial study, which identified multipotent precursor cells from adult mice pancreas that have the capacity to generate multiple pancreatic and neural cell types. ${ }^{29}$ We could perhaps extrapolate from thesefindings to explain the features seen in our case. It is possible that the hypoxia and structural aberrations induced by chemoradiation has triggered the tumour cells to exert its potential to proliferate along endocrine/neuroendocrine lines. This morphological appearance in the absence of staining for neuroendocrine and hormonal markers may suggest that the tumour was in a transitional phase. However, we concede that this suggestion is highly speculative and contentious at present. Increasing knowledge in the area of multipotent pancreatic precursor cells and pancreatic development may lead to a better understanding of pancreatic cancer and may pave the way for future therapeutic applications.

In summary, this overview provides a detailed morphological description of post-treated PDAC, highlighting several subtle and less-recognised findings. It is important for pathologists to be aware of these treatment-induced histopathological findings as NCRT for pancreatic cancer is becoming standard of care in many centres and also to avoid potential misinterpretation.

\section{Take home messages}

- Preoperative (neoadjuvant) chemoradiation therapy (NCRT) is emerging as an important treatment modality for borderline resectable pancreatic ductal adenocarcinoma (PDAC).

- NCRT can affect the morphological appearance of the tumour, stroma and non-neoplastic parenchyma which could potentially result in a diagnostic conundrum.

- The tumour shows a spectrum of changes including cytoplasmic eosinophilia, clear cell change and rarely oncocytic change and rhabdoid change.

- The tumour can rarely mimic non-neoplastic parenchyma (ie islet cells) and equally islet cells can arrange in "singlefiles" mimicking carcinoma or demonstrate perineural localisation, mimicking perineural invasion. 
Handling editor Cheok Soon Lee

Contributors The first and last authors (SNK and RC) contributed equally in the planning, conceptualising, conducting and writing of the manuscript. The second and third authors (SS and ND) contributed in the conducting of this review.

Competing interests None declared.

Provenance and peer review Not commissioned; externally peer reviewed.

\section{REFERENCES}

1 Evans DB, Rich TA, Byrd DR, et al. Preoperative chemoradiation and pancreaticoduodenectomy for adenocarcinoma of the pancreas. Arch Surg 1992:127:1335-9

2 Pisters PW, Abbruzzese JL, Janjan NA, et al. Rapid-fractionation preoperative chemoradiation, pancreaticoduodenectomy, and intraoperative radiation therapy for resectable pancreatic adenocarcinoma. J Clin Oncol 1998;16:3843-50.

3 Pisters PWT, Wolff RA, Janjan NA, et al. Preoperative paclitaxel and concurrent rapid-fractionation radiation for resectable pancreatic adenocarcinoma: toxicities, histologic response rates, and event-free outcome. J Clin Oncol 2002;20:2537-44.

4 Evans DB, Varadhachary GR, Crane $\mathrm{CH}$, et al. Preoperative gemcitabine-based chemoradiation for patients with resectable adenocarcinoma of the pancreatic head. J Clin Oncol 2008;26:3496-502.

5 Chatterjee D, Katz MH, Rashid A, et al. Histologic grading of the extent of residual carcinoma following neoadjuvant chemoradiation in pancreatic ductal adenocarcinoma: a predictor for patient outcome. Cancer 2012;118:3182-90.

6 Zhao Q, Rashid A, Gong Y, et al. Pathologic complete response to neoadjuvant therapy in patients with pancreatic ductal adenocarcinoma is associated with better prognosis. Ann Diagn Pathol 2012:16:29-37.

7 Hirata T, Teshima T, Nishiyama K, et al. Histopathologic tumor response after induction chemotherapy and stereotactic body radiation therapy for borderline resectable pancreatic cancer. Radiother Oncol 2015;13:1-7.

8 Mandard A-M, Dalibard F, Mandard J-C, et al. Pathologic assessment of tumor regression after preoperative chemoradiotherapy of esophageal carcinoma. Clinicopathologic correlations. Cancer 1994;73:2680-6.

9 Wheeler JM, Warren $\mathrm{BF}$, Jones $\mathrm{AC}$, et al. Preoperative radiotherapy for rectal cancer: implications for surgeons, pathologists and radiologists. Br J Surg 1999:86:1108-20.

10 West NP, Morris EJA, Rotimi 0, et al. Pathology grading of colon cancer surgical resection and its association with survival: a retrospective observational study. Lancet Oncol 2008;9:857-65.

11 Bouzourene $\mathrm{H}$, Bosman FT, Seelentag $\mathrm{W}$, et al. Importance of tumor regression assessment in predicting the outcome in patients with locally advanced rectal carcinoma who are treated with preoperative radiotherapy. Cancer 2002;94:1121-30.

12 Ryan R, Gibbons D, Hyland JMP, et al. Pathological response following long-course neoadjuvant chemoradiotherapy for locally advanced rectal cancer. Histopathology 2005;47:141-6.
13 Bartow SA, Mukai K, Rosai J. Pseudoneoplastic proliferation of endocrine cells in pancreatic fibrosis. Cancer 1981;47:2627-33.

14 Chatterjee D, Katz MH, Rashid A, et al. Pancreatic intraepithelial neoplasia and histological changes in non-neoplastic pancreas associated with neoadjuvant therapy in patients with pancreatic ductal adenocarcinoma. Histopathology 2013:63:841-51

15 Klimstra DS. Nonductal neoplasms of the pancreas. Mod Pathol 2007;20(Suppl 1): S94-112.

16 Dunne B, Reynolds JV, Mulligan E, et al. A pathological study of tumour regression in oesophageal adenocarcinoma treated with preoperative chemoradiotherapy. J Clin Pathol 2001;54:841-5.

17 Wang LM, Sheahan K. Pathological assessment of post-treatment gastrointestinal and hepatic resection specimens. Curr Diagn Pathology 2007:13:222-31.

18 Shimosato Y, Oboshi S, Baba K. Histologic evaluation of effects of radiotherapy and chemotherapy for carcinomas. Jpn J Clin Oncol 1971;1:19-35.

19 Wheeler JMD, Dodds E, Warren BF, et al. Preoperative chemoradiotherapy and total mesorectal excision surgery for locally advanced rectal cancer: correlation with rectal cancer regression grade. Dis Colon Rectum 2004;47:2025-31.

20 Hartman DJ, Krasinskas AM. Assessing treatment effect in pancreatic cancer. Arch Pathol Lab Med 2012;136:100-9.

21 Rouzbahman M, Serra S, Chetty R. Rectal adenocarcinoma with oncocytic features: possible relationship with preoperative chemoradiotherapy. J Clin Pathol 2006;59:1039-43.

22 Ambrosini-Spaltro A, Salvi F, Betts CM, et al. Oncocytic modifications in rectal adenocarcinomas after radio and chemotherapy. Virchows Arch 2006;448: 442-8.

23 Roy $\mathrm{P}$, Serra S, Kennedy E, et al. The prognostic value of grade of regression and oncocytic change in rectal adenocarcinoma treated with neo-adjuvant chemoradiotherapy. J Surg Oncol 2012;105:130-4.

24 Shia J, Tickoo SK, Guillem JG, et al. Increased endocrine cells in treated rectal adenocarcinomas: a possible reflection of endocrine differentiation in tumor cells induced by chemotherapy and radiotherapy. Am J Surg Pathol 2002;26:863-72.

25 Shia J, Guillem JG, Moore HG, et al. Patterns of morphologic alteration in residual rectal carcinoma following preoperative chemoradiation and their association with long-term outcome. Am J Surg Pathol 2004;28:215-23.

26 Evans AJ, Humphrey PA, Belani J, et al. Large cell neuroendocrine carcinoma of prostate: a clinicopathologic summary of 7 cases of a rare manifestation of advanced prostate cancer. Am J Surg Pathol 2006;30:684-93.

27 Klimstra DS, Rosai J, Heffess CS. Mixed acinar-endocrine carcinomas of the pancreas. Am J Surg Pathol 1994;18:765-78.

28 Furuyama $\mathrm{K}$, Kawaguchi $\mathrm{Y}$, Akiyama $\mathrm{H}$, et al. Continuous cell supply from a Sox9-expressing progenitor zone in adult liver, exocrine pancreas and intestine. Nat Genet 2011;43:34-41.

29 Seaberg RM, Smukler SR, Kieffer TJ, et al. Clonal identification of multipotent precursors from adult mouse pancreas that generate neural and pancreatic lineages. Nat Biotechnol 2004:22:1115-24. 